\title{
NEUT Pion FSI
}

\author{
Patrick de Perio \\ University of Toronto, Department of Physics, Toronto, Ontario, Canada
}

\begin{abstract}
The pion final state interaction model in NEUT is described. Modifications and tuning of the model are validated against neutrino and non-neutrino (pion scattering and photoproduction) data. A method for evaluating the uncertainties in the model and propagation of systematic errors in a neutrino oscillation experiment is described, using $\mathrm{T} 2 \mathrm{~K}$ as a specific example.
\end{abstract}

Keywords: NEUT, pion, nucleus, FSI, neutrino, T2K, ND280, Super-K, cross section, oscillation PACS: 25.20.Lj, 25.30.Pt, 25.80.Ek, 25.80.Gn, 25.80.Hp, 25.80.Ls

\section{INTRODUCTION}

Neutrino interaction generators play an integral role in neutrino-nucleus scattering and oscillation experiments. The vertex of a quasi-free interaction on a bound nucleon, assuming an impulse approximation, is referred to as the primary vertex. The final state hadrons produced at this vertex must propagate through the nucleus before observation in a detector. These particles can interact via the strong force, so there is a significant probability of re-interactions, or final state interactions (FSI), which affect the observable final state via absorption, scattering and particle production. This can obscure the true interaction mode of the primary vertex. Thus, recent experiments categorize measurements by the final state, instead of assuming some model to extract information about the primary vertex. This necessarily convolutes the physics of the primary vertex and FSI, allowing model builders to fit their own neutrino interaction and FSI models.

Our understanding of FSI feeds directly into neutrino oscillation experiments such as $\mathrm{T} 2 \mathrm{~K}$ [1]. The incident neutrino energy is required for precisely measuring oscillation parameters. A charged-current quasi-elastic (CCQE) measurement is useful since one can assume simple 2-body kinematics for reconstructing the neutrino energy from the outgoing lepton. However, $\mathrm{CC} 1 \pi$ interactions with $\pi$ absorption, can contaminate a CCQE measurement if the outgoing nucleons are undetectable, as is typically the case in Cerenkov detectors. The resulting energy spectrum would be distorted due to missing energy. Also, $\pi^{0}$ production in neutral current (NC) interactions can mimic the electromagnetic signature of an electron if one of the decay photons is not observed. This would contaminate a $v_{e}$ CCQE measurement for a $v_{e}$ appearance analysis. This motivates the need for an accurate FSI model that also has a good handle on systematic uncertainties.

This note describes recent modifications to the $\pi$ FSI model in the NEUT generator [2] that improve the agreement with $\pi$ scattering data. The data was then used for constraining model parameter uncertainties. A new reweighting method, which facilitates the propagation of these uncertainties into an oscillation analysis, is described. The new model and reweighting has been tested against SK atmospheric neutrino data and used in the $\mathrm{T} 2 \mathrm{~K} v_{e}$ appearance analysis [3]. 

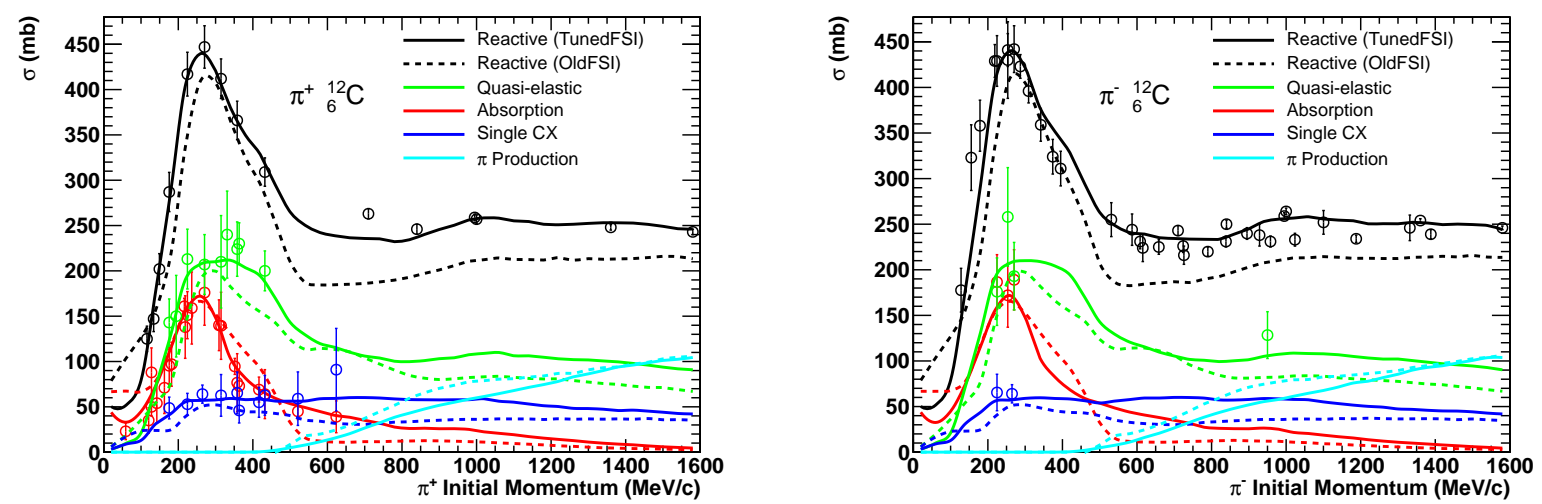

FIGURE 1. NEUT simulation of $\pi^{12} \mathrm{C}$ scattering compared to data from [e.g. 5, 6, 7, 8, 9]

\section{TUNING AND VALIDATION OF THE PION CASCADE MODEL}

The NEUT $\pi$ FSI model is a microscopic cascade that propagates the $\pi$ in finite steps through the nucleus. The mean free path (MFP) of various intranuclear mechanisms are calculated from a Delta-hole model [4] at low energy (LE), $p_{\pi} \leq 500 \mathrm{MeV} / \mathrm{c}$, and from free $\pi p$ scattering cross sections at high energy (HE), $p_{\pi}>500 \mathrm{MeV} / \mathrm{c}$. The MFPs are density (position) dependent and a Woods-Saxon distribution is assumed with parameters measured from electron scattering data. The microscopic method provides a connection between $\pi$ scattering measurements, where the $\pi$ originates from outside the nucleus, to neutrino experiments where the $\pi$ is produced within the nucleus. With $\pi$ scattering data, we can tune and constrain the FSI model, without the complication of any additional primary vertex physics.

At LE, the microscopic MFPs for absorption and scattering, were tuned from the original calculation so that the resulting cross sections agreed well with $\pi^{12} \mathrm{C}$ scattering data in Fig. 1. A "scattering" vertex within the nucleus can be either QE-like (same charge out) or single charge exchange (SCX), with the relative fraction determined by isospin. Note the simultaneous improved agreement in all channels.

At HE, the original NEUT model assumed an iso-scalar nucleus and defined the cross section (used for calculating the microscopic MFP) for scattering on a nucleon within the nucleus as: $\sigma_{Q E}=\frac{1}{2} \sigma_{\pi d}\left(\frac{\sigma_{Q E}^{\text {free }}}{\sigma_{\text {tot }}^{\text {free }}}\right)$, where $\sigma_{\pi d}$ is the total cross section for $\pi$ scattering on deuteron, and $\sigma_{Q E}^{\text {free }}$ and $\sigma_{\text {tot }}^{\text {free }}$ are the cross sections on free proton as shown in Fig. 2 . The $\pi^{+} p\left(\pi^{-} p\right)$ cross sections are also used for $\pi^{-} n\left(\pi^{+} n, \pi^{0} N\right)$ initial states, motivated by isospin symmetry. For hadron production: $\sigma_{\text {had }}=\frac{1}{2} \sigma_{\pi d}\left(1-\frac{\sigma_{Q E}^{\text {free }}}{\sigma_{\text {tot }}^{\text {free }}}\right)$.

The assumption of an iso-scalar nucleus breaks down when considering heavier nuclei. Hence, in the new model, the target nucleon is selected depending on the actual $p / n$ ratio of the nucleus, and the free $\pi^{ \pm} p$ cross sections are used directly as $\sigma_{Q E}=$ $\sigma_{Q E}^{\text {free }}, \sigma_{\text {had }}=\sigma_{\text {inel }}^{\text {free }}$ and (a new) $\sigma_{S C X}=\sigma_{S C X}^{\text {free }}$, as calculated by the SAID partial wave 

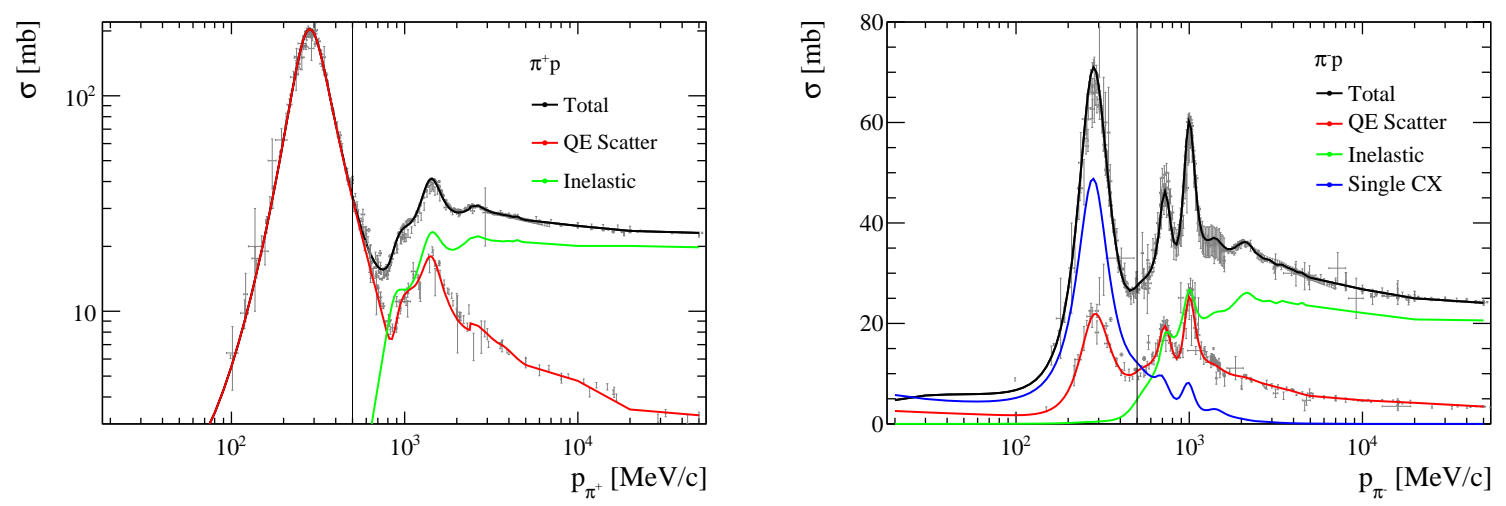

FIGURE 2. $\pi^{ \pm}$on free proton scattering cross sections. Data from the PDG and fits by SAID [10].
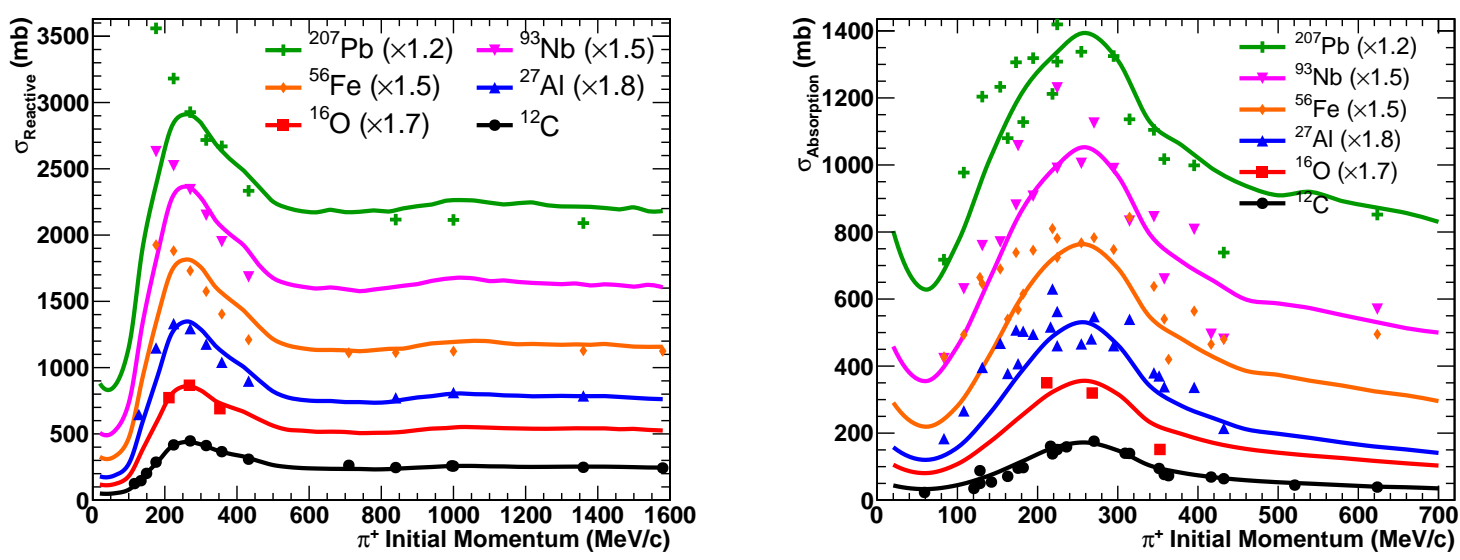

FIGURE 3. Reactive (left) and absorption (right) cross sections for various target nuclei (tuned FSI).
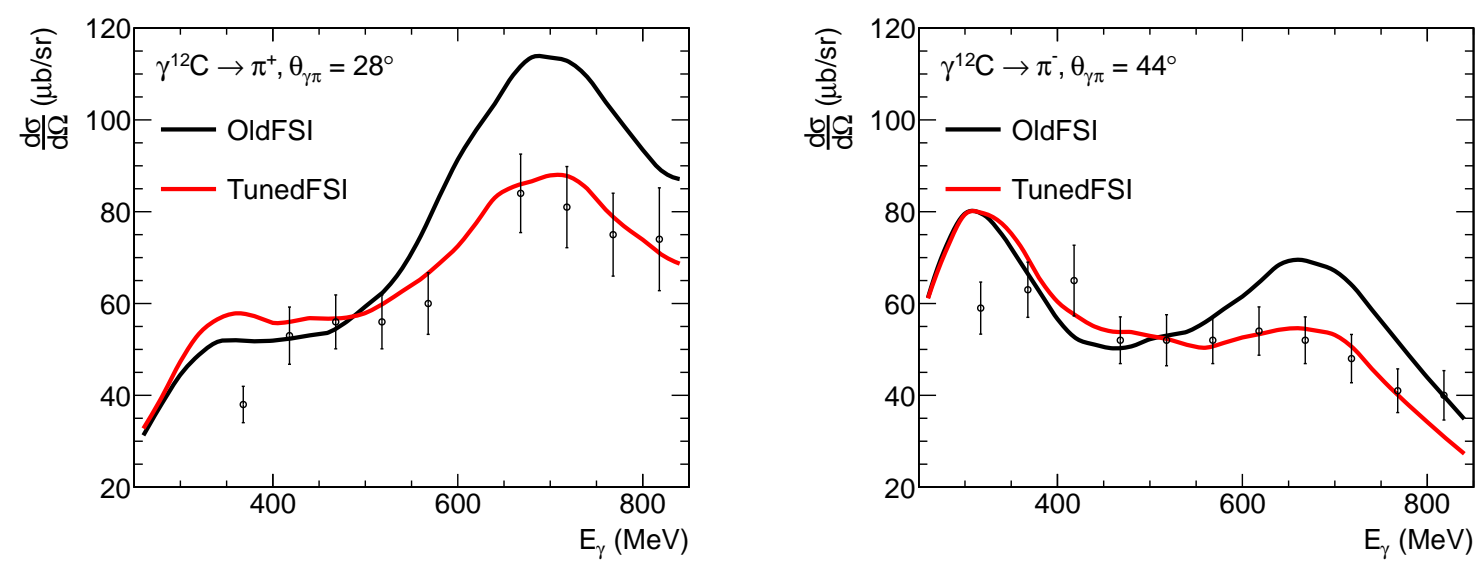

FIGURE 4. Differential cross sections for $\pi$ photoproduction off carbon, as a function of photon beam energy. Data points from [11]. 

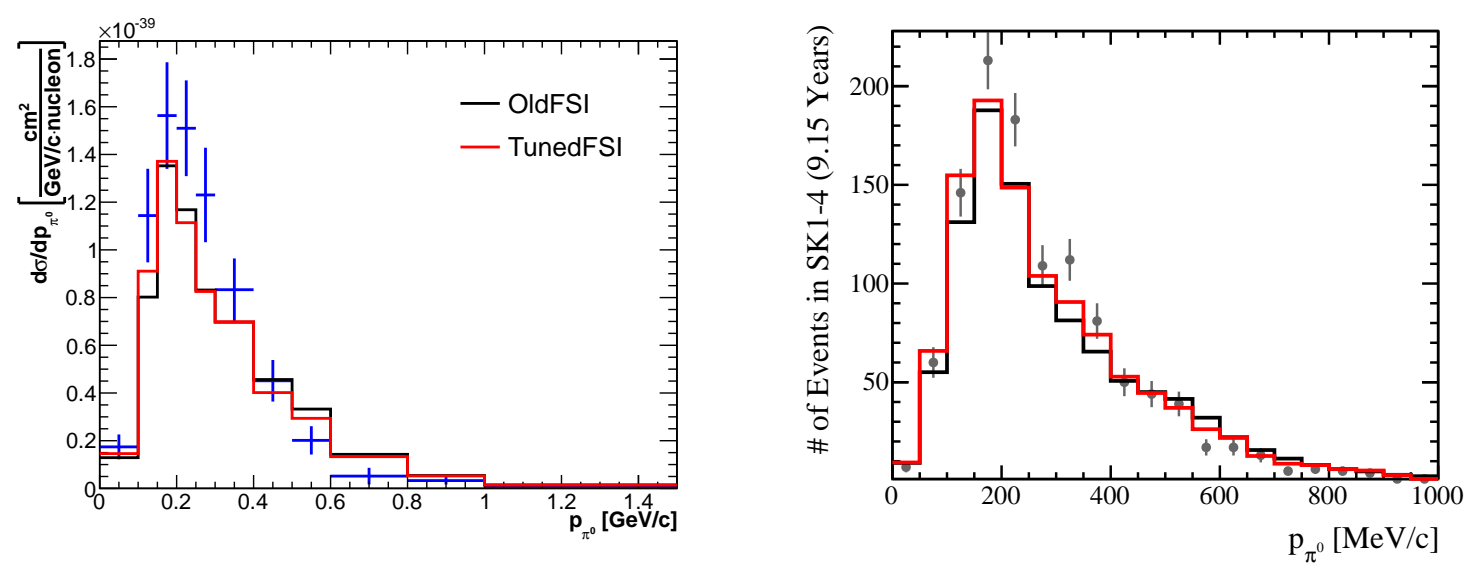

FIGURE 5. NEUT predictions for the MiniBooNE [15] (left) and SK (right, statistical error only) NC, combined-sign, $\pi^{0}$ measurements.

analysis (PWA) fit [10] shown in Fig. 2. This also takes into account the different energy dependencies of each interaction mechanism depending on the initial state. Finally, we scale both $\sigma_{Q E}$ and $\sigma_{S C X}$ by a constant factor of 1.8 , as motivated by $\pi^{12} \mathrm{C} \mathrm{QE}$ scattering [5] and SCX [6] data, similar to the scaling factor measured in proton-nucleus scattering [12].

The scattering kinematics at HE was originally determined from a simple elastic model: $\sin ^{2} \theta_{C M}=-\frac{q_{0}^{2} \log k}{4\left|\mathbf{p}_{\pi, \mathbf{C M}}\right|^{2}}$, where $q_{0}=200 \mathrm{MeV} / \mathrm{c}$ and $k$ is a random number. This model lacks backward scattering, and a deficit in absorption and SCX at HE compared to data in Fig. 1 also hints at this ${ }^{1}$. Hence, we implement the phase shifts from SAID [10] to calculate the differential cross section for HE $\pi N$ scattering in the new model.

The modifications to the HE region result in better agreement with data in all channels as shown in Fig. 1. Blending of the LE and HE model was implemented to improve continuity at the boundary. Pion scattering with heavier targets was also checked and we observe accurate reproduction of the $A$ dependence in each interaction channel as shown in Fig. 3, simply by varying the nuclear size and density distribution in the simulation. This provides confidence in the FSI model when simulating neutrino interactions on heavy nuclei, as in the T2K near detector (ND280) or the surrounding rock of SK. We expect $95 \%$ of $\pi$ produced with the T2K neutrino beam to have momenta $<2 \mathrm{GeV} / \mathrm{c}$.

A $\pi$-photoproduction simulation was developed, similar to that used in [13], where the final state kinematics of the primary vertex are determined from a PWA fit of free nucleon photoproduction data [14]. The results are compared to total, differential and double differential cross section data on carbon $[11,13]$. For $E_{\gamma}>500 \mathrm{MeV}$, the addition of backward scattering to the FSI model, also resulting in more absorption, decreases the calculated cross section for forward going $\pi$ bringing the simulation into agreement with the data in Fig. 4.

\footnotetext{
${ }^{1}$ More backward scattering would tend to reduce the $\pi$ energy into the $\Delta$ resonance region.
} 
Finally, the full NEUT neutrino simulation was used with the MiniBooNE (MB) flux on $\mathrm{CH}_{2}$ and compared to all available $\mathrm{MB}$ cross section data [e.g. 15]. Atmospheric neutrino interactions on $\mathrm{H}_{2} \mathrm{O}$ at $\mathrm{SK}$ were also simulated, including the detector response and reconstruction [1]. In Fig. 5, we select NC events with a single outgoing $\pi^{0}$ for the MB comparison, and for SK, events with 2 electron-like rings with reconstructed invariant mass between 85 and $185 \mathrm{MeV} / \mathrm{c}$ and no decay electron. There is slightly better agreement in the shape and absolute normalization after the FSI modifications.

\section{FSI CASCADE REWEIGHTING AND UNCERTAINTIES}

Proper treatment of FSI systematics in neutrino experiments has been a longstanding problem. Variations in the final state would require regeneration of detector $\mathrm{MC}$ and reconstruction, a very CPU intensive task. To more easily predict changes in detector observables resulting from variations in microscopic MFPs, a reweighting scheme was developed which preserves the details of the microscopic cascade and correlations between the different intranuclear mechanisms.

For each MC event we store the following truth information: $\pi$ starting and exit position, position of each FSI vertex, mechanism at each vertex, $\pi$ type and momentum between each vertex and nucleus type. With this information, we can rerun the same cascade using the exact trajectory to calculate the probability $P_{\text {evt }}$ for the event. The survival probability in a given step of the cascade is: $P_{\text {surv }}(\mathbf{r}, h, p)=1-\sum_{i} P_{i}(\mathbf{r}, h, p)$, where $\mathbf{r}$ is the current position within the nucleus, $h$ is the $\pi$ type, $p$ is the momentum and $i$ denotes the various interaction mechanisms. Then the probability for this given trajectory is: $P_{\text {traj }}=\prod_{\text {all steps }} P_{\text {step }}$, where $P_{\text {step }}=P_{\text {surv }}$ or $P_{i}$ depending on what occurred in the given step. The probability for an event with multiple $\pi$ is then: $P_{\text {evt }}=\prod_{\text {all trajs }} P_{\text {traj }}$.

We define a set of energy and position independent scaling parameters, $f_{i}$, which scale each interaction probability, $P_{i}$. The modified probability of interaction for a given step is then $P_{i}^{\prime}=f_{i} P_{i}$ which is used to recalculate $P_{\text {evt }}$. Finally, the weight for an event given a set of modified $f_{i}$ is: $w_{\text {evt }}\left(f_{i}\right)=\frac{P_{\text {evt }}^{\prime}\left(f_{i}\right)}{P_{\text {evt }}}$. This weight can be used when generating detector observable distributions, to observe the effect of varying the FSI model.

Three LE scaling parameters were simultaneously fitted to $\pi^{12} \mathrm{C}$ data and the resulting eight $1 \sigma$ parameter sets (one from each octant of the 3-parameter space) are shown in Fig. 6 (left). This set of parameters conservatively spans the error of the data and is propagated through the T2K-SK and ND280 MC. The scaling parameters for HE were also varied for each LE set to maximize and minimize the event multiplicity. Furthermore, we vary the secondary interaction cross sections in the SK detector simulation in a correlated manner with the intranuclear variations. For the T2K $v_{e}$ appearance analysis [3], we simply use the maximum deviation across all FSI variations as the $1 \sigma$ systematic error due to FSI uncertainties, as shown in Fig. 6 (right). The increase in error towards low neutrino energy is due to non-CCQE events with all pions absorbed. For the ND280 CC inclusive measurement in [3], the effect is less significant $(<1 \%)$ since it is insensitive to FSI. However, future exclusive selections will benefit from this reweighting scheme. Also, future oscillation analyses will be able to properly handle correlations introduced by FSI uncertainties between near and far detectors. 

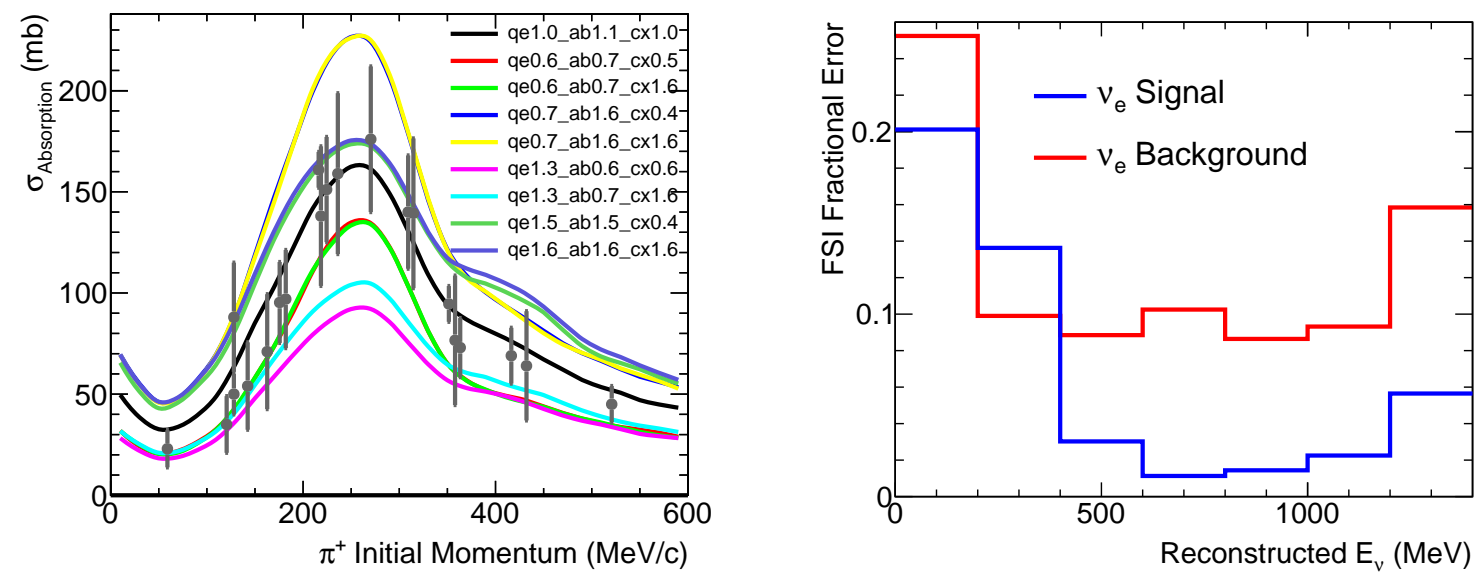

FIGURE 6. Left: Tuned (black) and LE parameter variation $1 \sigma$ curves from simultaneous fit to $\pi$ scattering data. Right: Maximum deviation of the LE and HE FSI variations for the T2K-SK $v_{e}$ appearance (CCQE-like) sample, where " $v_{e}$ Signal" comes from CC interactions of $v_{e}$ oscillated from $v_{\mu}$ [3].

\section{SUMMARY AND ACKNOWLEDGMENTS}

Modifications to the NEUT FSI model, necessary for extracting a constraint from $\pi$ scattering data, were described. The model shows good agreement compared to external pion scattering, photoproduction and neutrino data. A reweighting scheme was developed for evaluating systematic errors due to FSI uncertainties, with an example application to the $\mathrm{T} 2 \mathrm{~K}$ experiment.

I am grateful for the tutelage of my colleagues at Super-Kamiokande, especially Yoshinari Hayato. I acknowledge the support of the NSERC MSFSS, CGS and Vanier programs.

\section{REFERENCES}

1. K. Abe, N. Abgrall, H. Aihara, et al., Nucl. Instr. Meth. A 659, 106 - 135 (2011).

2. Y. Hayato, Acta Physica Polonica B 40, 2477 (2009).

3. K. Abe, N. Abgrall, Y. Ajima, et al., Phys. Rev. Lett. 107, 041801 (2011).

4. L. Salcedo et al., Nucl. Phys. A 484, 557 (1988).

5. Y. Fujii et al., Phys. Rev. C 64, 034608 (2001).

6. M. K. Jones et al., Phys. Rev. C 48, 2800 (1993).

7. D. Ashery et al., Phys. Rev. C 23, 2173 (1981).

8. B. W. Allardyce et al., Nucl. Phys. A 209, 1 (1973).

9. T. Lee et al., Annu. Rev. Nucl. 52, 23-63 (2002).

10. R. Arndt et al., Phys. Rev. C 69, 035213 (2004).

11. K. Baba et al., Nucl. Phys. A 306, 292 (1978).

12. G. Bellettini et al., Nucl. Phys. 79, 609 (1966).

13. J. Arends et al., Z. Phys. A 305, 205 (1982).

14. R. Arndt et al., Phys. Rev. C 66, 055213 (2002).

15. A. A. Aguilar-Arevalo, C. E. Anderson, A. O. Bazarko, et al., Phys. Rev. D 81, 013005 (2010). 